\title{
Effects of CPAP therapy withdrawal on exhaled breath pattern in obstructive sleep apnoea
}

\author{
Esther I Schwarz, ${ }^{1}$ Pablo Martinez-Lozano Sinues, ${ }^{2}$ Lukas Bregy, ${ }_{1}^{2}$ Thomas Gaisl, ${ }^{1}$ \\ Diego Garcia Gomez, ${ }^{2}$ Martin T Gaugg, ${ }^{2}$ Yannick Suter, ${ }^{2}$ Nina Stebler, ${ }^{1}$ \\ Yvonne Nussbaumer-Ochsner, ${ }^{1}$ Konrad E Bloch, ${ }^{1,3,4}$ John R Stradling, ${ }^{5}$ \\ Renato Zenobi, ${ }^{2}$ Malcolm Kohler ${ }^{1,3,4}$
}

\begin{abstract}
- Additional material is published online only. To view please visit the journal online (http://dx.doi.org/10.1136/ thoraxjnl-2015-207597).
\end{abstract}

${ }^{1}$ Department of Pulmonology and Sleep Disorders Centre, University Hospital of Zurich Zurich, Switzerland

${ }^{2}$ Department of Chemistry and Applied Biosciences, Swiss Federal Institute of Technology, Zurich, Switzerland

${ }^{3}$ Centre for Integrative Human Physiology, University of Zurich, Zurich, Switzerland

${ }^{4}$ Zurich Centre for

Interdisciplinary Sleep Research, University of Zurich, Zurich, Switzerland

${ }^{5}$ Oxford Centre for Respiratory Medicine and NIHR Oxford Biomedical Research Centre, Churchill Hospital, Oxford, UK

\section{Correspondence to}

Professor Malcolm Kohler, Chair Respiratory Medicine, Clinical Director, Department of Pulmonology and Sleep Disorders Centre, University Hospital of Zurich,

Raemistrasse 100,

Zurich CH-8091, Switzerland; malcolm.kohler@usz.ch

*EIS and PM-LS contributed equally to this paper.

Received 25 July 2015 Revised 10 November 2015 Accepted 16 November 2015 Published Online First 15 December 2015

\section{ABSTRACT}

Background Obstructive sleep apnoea (OSA) is highly prevalent and associated with cardiovascular and metabolic changes. OSA is usually diagnosed by polysomnography which is time-consuming and provides little information on the patient's phenotype thus limiting a personalised treatment approach. Exhaled breath contains information on metabolism which can be analysed by mass spectrometry within minutes. The objective of this study was to identify a breath profile in OSA recurrence by use of secondary-electrosprayionization-mass spectrometry (SESI-MS).

Methods Patients with OSA effectively treated with CPAP were randomised to either withdraw treatment (subtherapeutic (PAP) or continue therapeutic CPAP for 2 weeks. Exhaled breath analysis by untargeted SESI-MS was performed at baseline and 2 weeks after randomisation. The primary outcome was the change in exhaled molecular breath pattern.

Results 30 patients with OSA were randomised and 26 completed the trial according to the protocol. CPAP withdrawal led to a recurrence of OSA (mean difference in change of oxygen desaturation index between groups $+30.3 / \mathrm{h} ; 95 \% \mathrm{Cl} 19.8 / \mathrm{h}, 40.7 / \mathrm{h}, \mathrm{p}<0.001)$ which was accompanied by a significant change in 62 exhaled features (16 metabolites identified). The panel of discriminating mass-spectral features allowed differentiation between treated and untreated OSA with a sensitivity of $92.9 \%$ and a specificity of $84.6 \%$.

Conclusion Exhaled breath analysis by SESI-MS allows rapid and accurate detection of OSA recurrence. The technique has the potential to characterise an individual's metabolic response to OSA and thus makes a comprehensible phenotyping of OSA possible.

Trial registration number NCT02050425 (registered at ClinicalTrials.gov).

\section{INTRODUCTION}

Obstructive sleep apnoea (OSA) is a highly prevalent and underdiagnosed sleep-related breathing disorder commonly associated with daytime sleepiness, increased rate of accidents, vascular dysfunction and hypertension, adverse cardiovascular outcome and diverse metabolic changes. ${ }^{1-6}$ The major biological mechanisms underpinning the association between OSA and its systemic pathophysiological consequences are thought to include apnoea-related intermittent hypoxia leading to

\section{Key messages}

\section{What is the key question?}

- The question was brought up whether a disease-specific breath profile of obstructive sleep apnoea (OSA) giving insight into the systemic and metabolic consequences of OSA can be detected by the novel approach of breath analysis using untargeted real-time mass spectrometry.

\section{What is the bottom line?}

- This randomised controlled trial is the first study providing data on metabolic breath-prints using untargeted real-time mass spectrometry in OSA and shows that real-time breath analysis by mass spectrometry allows accurate detection of untreated OSA within minutes.

\section{Why read on?}

- The findings of this study provide a basis for future research using the non-invasive approach of breath analysis allowing the identification of specific diseases such as OSA, novel disease mediators or markers that may serve as both diagnostic and therapeutic targets, and could be translated into clinical practice.

increased oxidative stress and increased sympathetic activity and arousal-induced reflex sympathetic activation. $^{7}$

The gold-standard diagnostic test for OSA is in-laboratory polysomnography. Alternatively, portable home monitoring devices can diagnose OSA with sufficient accuracy in subjects with a high pre-test probability for OSA. However, all sleep studies are time-consuming and costly, and sometimes inconclusive due to technical artefacts or impaired sleep in an unaccustomed environment. Moreover, usual measures of disease severity derived from sleep studies do not allow reliable differentiation between different phenotypes of OSA, that is, cannot identify subjects who are susceptible to metabolic, neurological and cardiovascular effects of OSA.

OSA can be effectively treated with nocturnal CPAP which abolishes apnoea and hypopnoea and thus prevents the pathophysiological consequences 
of OSA. Short-term CPAP therapy withdrawal, in patients hitherto compliant with CPAP, can be applied to efficiently investigate the effects of OSA recurrence on physiology. ${ }^{4}$

Exhaled breath contains biochemical information about metabolism and its alterations. Most of the numerous exhaled compounds in breath are not produced in the lungs but released along the blood-gas barrier during gas exchange. Metabolic alterations are reflected in perturbed metabolic profiles that may be used to support the diagnosis of diseases such as OSA. This hypothesis is supported by studies suggesting that sleep apnoea results in altered plasma metabolites. ${ }^{8}$ The main drawback of traditional plasma-based metabolomic analyses is that these procedures are time-consuming and labour-intensive. Alternatively, the wealth of metabolic information contained in exhaled breath could be analysed using electronic sensors. ${ }^{9}{ }^{10}$ However, the poor chemical specificity of electronic sensors does not allow for structural elucidation of the molecules imparting such OSA-specific breath-prints, limiting the usefulness of this technology. In this study, an approach combining the analytical power of mass spectrometry with the speed and noninvasiveness of electronic sensors was for the first time applied in patients with OSA to provide a comprehensive analysis of exhaled breath metabolites in real time. This technique, dubbed secondary electrospray ionisation-mass spectrometry (SESI-MS), has shown promise in detecting a wide range of metabolites in breath and allows rapid screening of patients. ${ }^{11-16}$

The objective of the study was to answer the question whether there is a disease-specific profile of exhaled breath in patients with OSA applying untargeted mass spectrometry, which might be used to confirm the diagnosis and also provide insight into the pathophysiology of OSA and underlying disease mechanisms on a metabolic level.

\section{METHODS}

Trial design

Randomised controlled parallel group trial.

\section{Subjects}

Patients aged between 20 and 75 years with known moderate-to-severe OSA with an oxygen desaturation index (ODI) of $>20 / \mathrm{h}$ at the time of diagnosis were eligible if they currently had an ODI of $>20 / \mathrm{h}$ ( $\geq 4 \%$-dips) during an ambulatory nocturnal pulse oximetry on the fourth night off CPAP, and were effectively treated with CPAP for $>12$ months $^{4}{ }^{17}$ (see online supplementary file).

\section{Sample size}

Because a formal power calculation based on the primary outcome was not possible, sample size estimation was based on the assumption that a clinically relevant difference in ODIreflecting the disease of interest-between therapeutic and subtherapeutic CPAP is $15 / \mathrm{h}$ (SD12). ${ }^{4}{ }^{17}$ Based on this assumption, power calculation indicated that 28 patients were required in total to not miss a clinically relevant difference in ODI with a power of $90 \%(\alpha=0.05)$ (see online supplementary file).

\section{Patient evaluation and follow-up}

The study was conducted at the University Hospital Zurich and Swiss Federal Institute of Technology, Zurich, Switzerland. The first patient was recruited in February 2014 and the last patient follow-up was completed in August 2014. Patients with OSA effectively treated with CPAP were randomly assigned (allocation ratio $1: 1)$ to one of the two treatment groups-either to continue therapeutic CPAP or to withdraw it by the use of a subtherapeutic device. Follow-up assessments were performed 2 weeks after randomisation. Allocation was determined by unsorted envelopes. A member of the team not involved in patient enrolment and outcome assessment generated the allocation sequence and prepared the CPAP devices. Patients remained blinded to allocation.

The study protocol was approved by the cantonal ethics committee of Zurich (KEK-ZH-Nr.2013-0536). The trial was conducted according to the Declaration of Helsinki and registered at ClinicalTrials.gov (NCT02050425). Written informed consent was obtained from participants before participation in the trial.

\section{Sleep studies and CPAP devices}

Home sleep studies using the ApneaLink Plus-device (ResMed, San Diego, USA) were performed the night before the baseline and the follow-up breath analysis. OSA severity was quantified by ODI ( $\geq 4 \%$-dips). Recurrence of OSA was defined as an ODI of $>15 / \mathrm{h}$ in the follow-up sleep study (see online supplementary file).

\section{Primary outcome}

The primary outcome was the change in exhaled breath pattern in response to CPAP withdrawal (exhaled breath pattern in OSA recurrence) and its diagnostic accuracy.

Real-time breath analysis was performed using SESI-MS ${ }^{14}$ coupled to a quadrupole time-of-flight mass spectrometer (AB Sciex, Concord, Canada) (see online supplementary file).

\section{Secondary outcome measures}

Secondary outcomes were changes in ODI, relationship between change in breath signal intensity and ODI and the chemical identification of the compounds differentiating treated from untreated OSA (see online supplementary file).

\section{Data analysis}

Changes within and between groups were analysed by dependent and independent $t$ test, respectively. In all cases, 5000 bootstrap samples were used to compute $\mathrm{p}$ values. Subsequent estimate of the false discovery rate for multiple hypothesis testing was performed by computing $q$ values for each $\mathrm{p}$ value as instructed by Storey. ${ }^{18}$ Statistical significance level was set to $\alpha<0.05$. Pearson's linear correlation coefficient and $95 \%$ bootstrap CIs between changes in breath signal intensity and changes in ODI were computed. The most correlating features were subjected to principal component analysis (PCA). OSA prediction was accomplished in a leave-one-out-cross-validation (LOOCV) by feature selection and subsequent classification using a random forest classification algorithm. ${ }^{19}$ The most discriminative features were used to compute hierarchical cluster analysis and multidimensional scaling of the proximity matrix.

Statistica (V6.0; StatSoft, Tulsa, USA) and Matlab (MathWorks, Natick, USA) were used for data analysis. Further details on data analysis procedures are described in the online supplementary file.

\section{RESULTS}

\section{Trial profile and patient characteristics}

The study flow is shown in figure 1. Thirty patients with moderate-to-severe OSA were randomly assigned to either continue therapeutic CPAP or to withdraw it by the use of a subtherapeutic CPAP device. Of the30 patients, 28 (14 in each treatment group) completed the study. One participant in each group was lost to follow-up: one patient randomised to 
Figure 1 Consort diagram.

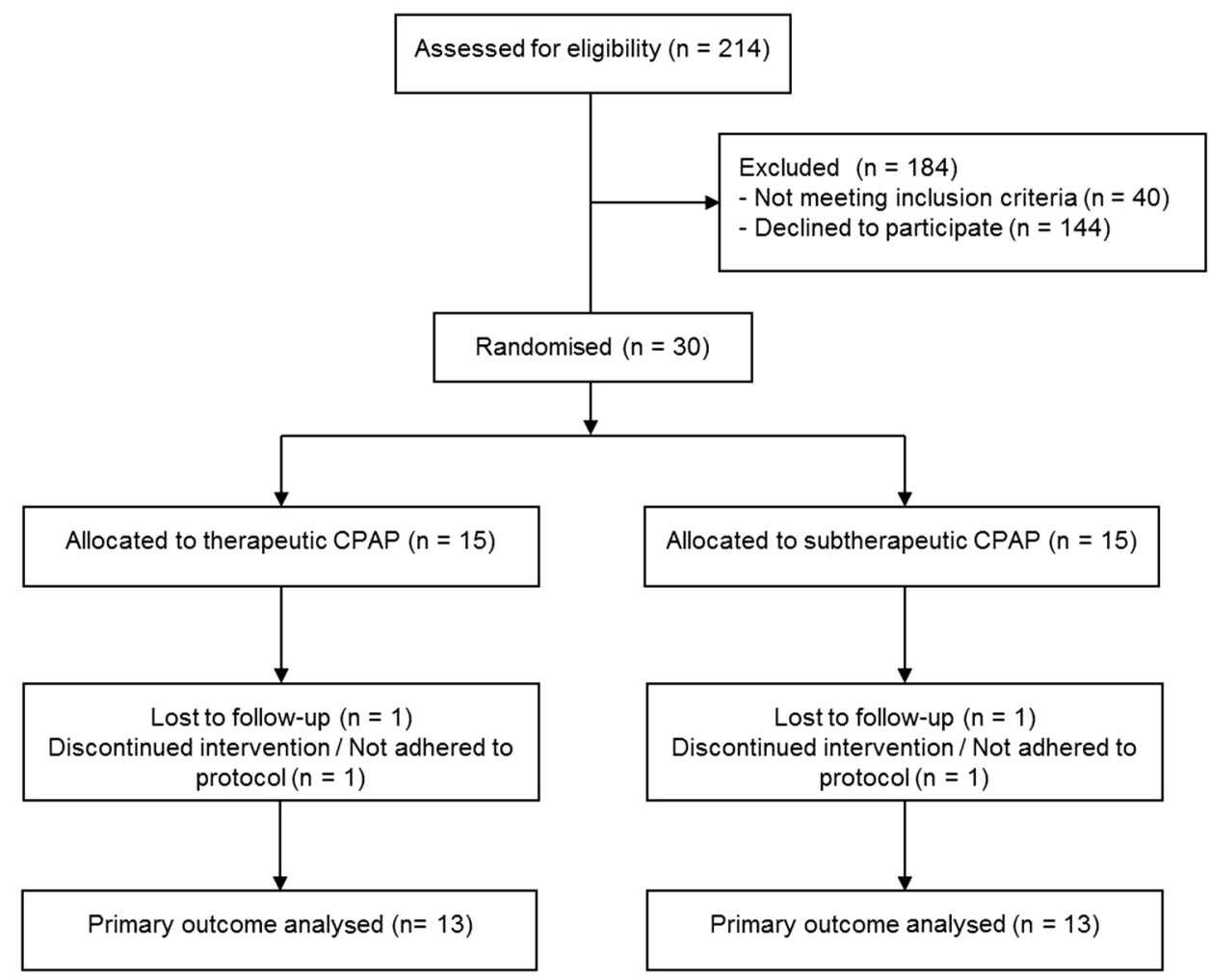

therapeutic CPAP did not show up to the follow-up visit and withdrew from the study due to personal reasons, and one subject allocated to subtherapeutic CPAP was lost to follow-up due to a relapse of a major depression requiring hospitalisation. Two subjects did not adhere to the protocol: one participant randomised to therapeutic CPAP experienced difficulties handling the provided CPAP device and did not use it consistently thus having relevant OSA at follow-up, and one participant randomised to subtherapeutic CPAP manipulated his sham device.

Table 1 Baseline patient characteristics

\begin{tabular}{lll}
\hline & $\begin{array}{l}\text { Therapeutic } \\
\text { CPAP } \\
(\mathbf{n}=13)\end{array}$ & $\begin{array}{l}\text { CPAP- } \\
\text { withdrawal } \\
(\mathbf{n}=13)\end{array}$ \\
\hline Age, mean (SD), years & $65.3 \pm 7.7$ & $67.6 \pm 6.5$ \\
Male sex, n (\%) & $11(85)$ & $9(69)$ \\
BMI, mean (SD), kg/m ${ }^{2}$ & $32.8 \pm 5.4$ & $34.4 \pm 6.2$ \\
Neck circumference, mean (SD), cm & $44.2 \pm 3.4$ & $40.2 \pm 3.4$ \\
Active smoker, n (\%) & $1(8)$ & $1(8)$ \\
Former smoker, n (\%) & $3(23)$ & $8(62)$ \\
Antihypertensive drugs, n (\%) & $5(38)$ & $9(69)$ \\
Statins, n (\%) & $7(54)$ & $4(31)$ \\
AHI at diagnosis, mean (SD), events per hour & $55.3 \pm 22.6$ & $45.8 \pm 16.4$ \\
ODI at diagnosis, mean (SD), events per hour & $53.0 \pm 20.8$ & $42.6 \pm 17.4$ \\
ESS at diagnosis, mean (SD), points & $13.0 \pm 2.9$ & $12.3 \pm 4.8$ \\
AHI on CPAP, mean (SD), events per hour & $4.1 \pm 3.0$ & $2.1 \pm 1.5$ \\
ESS on CPAP, mean (SD), points & $5.1 \pm 2.2$ & $6.5 \pm 2.5$ \\
CPAP compliance, mean (SD), hh:mm & $06: 50$ & $07: 08$ \\
FEV ${ }_{1} /$ FVC, mean (SD) & $0.80(0.04)$ & $0.78(0.02)$ \\
FVC, mean (SD), \% predicted & $90(9)$ & $95(16)$ \\
FEV ${ }_{1}$, mean (SD), \% predicted & $89(13)$ & $95(16)$ \\
\hline
\end{tabular}

AHI, apnoea-hypopnoea-index; BMI, body mass index; ESS, Epworth Sleepiness Scale; ODI, oxygen desaturation-index.
Thus, the primary outcome was analysed in 26 patients who completed the trial per protocol. Baseline data are shown in table 1.

\section{Effects of CPAP withdrawal on OSA}

CPAP withdrawal was associated with return of OSA as evidenced by a significant increase in ODI (mean difference in change between groups, $+30.3 / \mathrm{h}, 95 \% \mathrm{CI}+19.8$ to $+40.7 / \mathrm{h}$, $\mathrm{p}<0.001$ ) (table 2).

\section{Effects of CPAP withdrawal on exhaled breath \\ Exhaled breath pattern (primary outcome)}

When compared with continuing CPAP, withdrawal of CPAP, and thus recurrence of OSA, was associated with altered exhaled breath patterns as determined by a statistically significant increase in 61 features and a decrease in 1 feature (see online supplementary table E1). Figure $2 \mathrm{~A}-\mathrm{E}$ shows one exemplary molecule which significantly increased in response to CPAP withdrawal. Online supplementary figure E3 shows additional examples of metabolites which allowed distinguishing between treated and untreated OSA. A blind prediction of OSA recurrence (defined as ODI $>15 / \mathrm{h}$ ) by a LOOCV using the 15 most-discriminating exhaled features in each cross-validation resulted in a sensitivity of $92.9 \%$ and specificity of $84.6 \%$ (table 3 and receiver operating characteristic (ROC) curve in figure $3 \mathrm{~A})$. The area under the ROC curve was 0.874 (95\% CI 0.612 to 1 ). How these features discriminate the two groups is visualised in figure $3 \mathrm{~B}, \mathrm{C}$.

\section{Chemical identification of exhaled breath compounds}

A comprehensive structural elucidation strategy allowed chemical identification of 22 molecules in the breath of patients with OSA (see eg, benzothiazole in figure 3D). Of the 22 identified molecules, 16 were shown to significantly change in response to CPAP withdrawal. These molecules are listed in table 4 (see also 
Table 2 Outcomes from sleep studies

\begin{tabular}{|c|c|c|c|c|c|c|c|}
\hline & \multicolumn{2}{|c|}{ Therapeutic CPAP $(n=13)$} & \multicolumn{2}{|c|}{ Subtherapeutic CPAP $(n=13)$} & \multicolumn{3}{|l|}{ Treatment effect } \\
\hline & Baseline & follow-up & Baseline & Follow-up & Difference in change & $95 \% \mathrm{Cl}$ & p Value \\
\hline ODI & $4.3(3.2)$ & $4.2(1.7)$ & $3.9(3.5)$ & $34.0(16.3)$ & +30.3 & +19.8 to +40.7 & $<0.001$ \\
\hline
\end{tabular}

online supplementary tables E1 and E3). Most of the identified molecules stem from a cluster of chemical families (eg, aldehydes). There is a strong association among the molecules within each chemical family (see figure $3 \mathrm{E}$ and online supplementary figure E6).
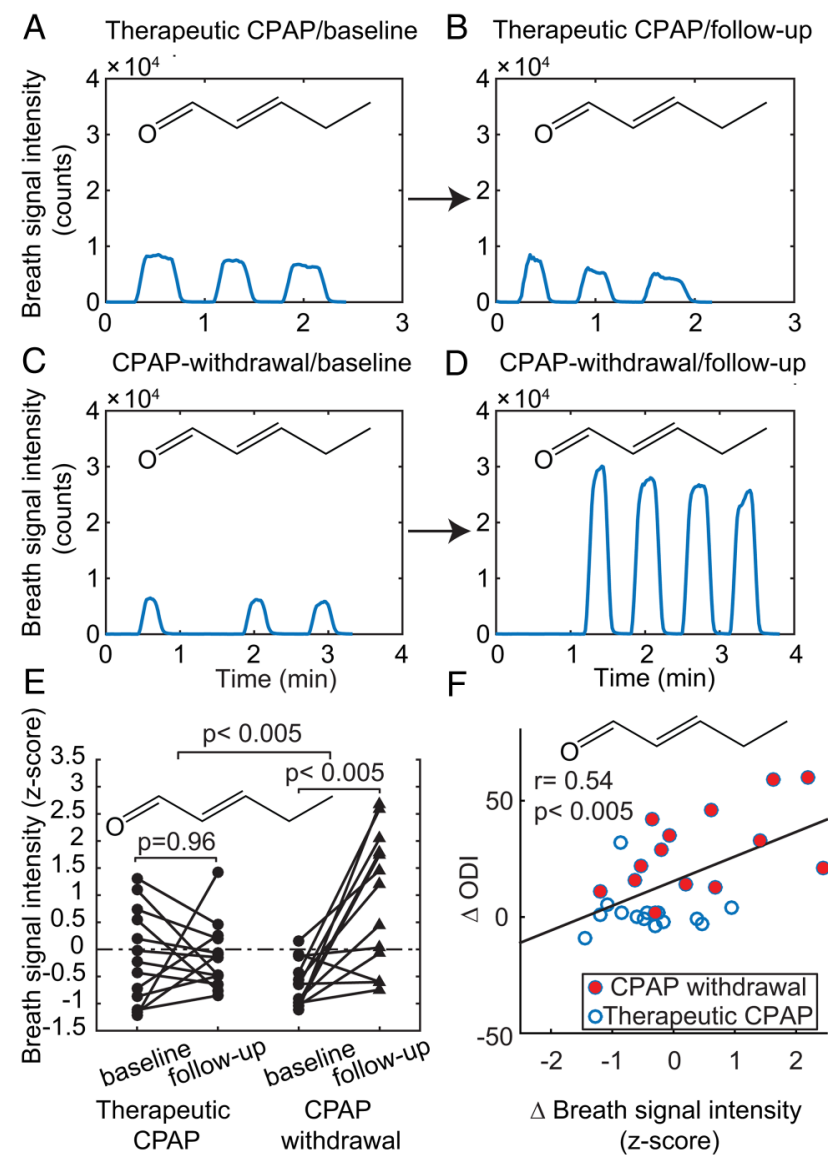

Figure 2 Pentenal in exhaled breath. Real-time breath analysis showing time traces of the signal intensity of one exemplary breath metabolite (pentenal) in a subject from the therapeutic CPAP and a subject from the CPAP-withdrawal group at baseline and at follow-up (A-D). The smoothed signals intensity traces increase during the exhalation cycles, providing good repeatability in signal intensity among the three to four replicate exhalations recorded within minutes. Signal intensity in the patient on therapeutic CPAP does not change from baseline (A) to follow-up (B) $\left(7.4 \times 10^{3}\right.$ to $5.7 \times 10^{3}$ counts). In contrast, the patient undergoing CPAP withdrawal showed a signal increase from $6 \times 10^{3}(\mathrm{C})$ counts at baseline to $2.7 \times 10^{4}$ (D) counts at follow-up (ie, 4.5-fold increase). Individual plots (E) of 2-pentenal in both groups $(n=26)$ show significant breath compound enhancement. Correlation $(F)$ is shown between changes in breath signal intensity of pentenal and changes in oxygen desaturation index $(n=28)(r=0.54$; $95 \% \mathrm{Cl} 0.2$ to $0.78 ; \mathrm{p}<0.003)$.
Association of breath signals with sleep apnoea severity

In addition to identification of metabolites changing in response to CPAP withdrawal, those showing a significant association between changes in signal intensity and disease severity were identified. Fifty-four features correlated significantly with changes in ODI as a measure of disease severity (see online supplementary table E2), including all 16 identified metabolites constituting the disease-specific breath profile (see figure $2 \mathrm{~F}$ and online supplementary figure E4). The overall correlation of these compounds with disease severity is visualised in online supplementary figure E5 by reducing the significantly correlating features to one dimension using PCA.

\section{DISCUSSION}

This is the first study applying untargeted mass spectrometry to extract information from the exhaled breath metabolome in patients with OSA and paving the way for identifying a diseasespecific breath profile. The use of the CPAP therapy withdrawal trial design enabled us to study OSA-specific breath patterns in a randomised controlled fashion, thus avoiding the possible biases of observational case-control studies.

The gold standard for OSA diagnosis, that is, in-laboratory polysomnography, is technically demanding and timeconsuming. In addition, measures of OSA severity derived from sleep studies do not differentiate between distinct phenotypes of OSA, for example, symptomatic subjects or subjects with a pronounced increase in sympathetic activity or oxidative stress and thus increased vascular risk. For these reasons, a number of ongoing studies are attempting to define markers of sleep disorders at the protein and metabolic levels. ${ }^{20} 21$ In contrast to blood and tissue specimens, exhaled breath carries easily accessible molecular markers, some of which are released along the blood-gas barrier and thus reflect changes in the blood and cell environment. It has been suggested that metabolites might move from different compartments into the blood and are then released via breath. ${ }^{22}$

Up to now there are only limited data in OSA derived from a small number of pilot studies on exhaled breath using electronic

Table 3 Diagnostic accuracy of exhaled breath analysis in OSA

\begin{tabular}{|c|c|c|c|}
\hline & \multicolumn{2}{|c|}{ Predicted } & \multirow[b]{2}{*}{ Total } \\
\hline & OSA & No OSA & \\
\hline \multicolumn{4}{|l|}{ Condition } \\
\hline OSA & 13 & 1 & 14 \\
\hline No OSA & 2 & 11 & 13 \\
\hline Total & 15 & 12 & 27 \\
\hline
\end{tabular}




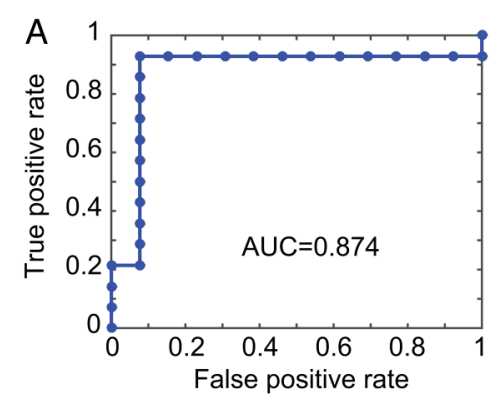

C

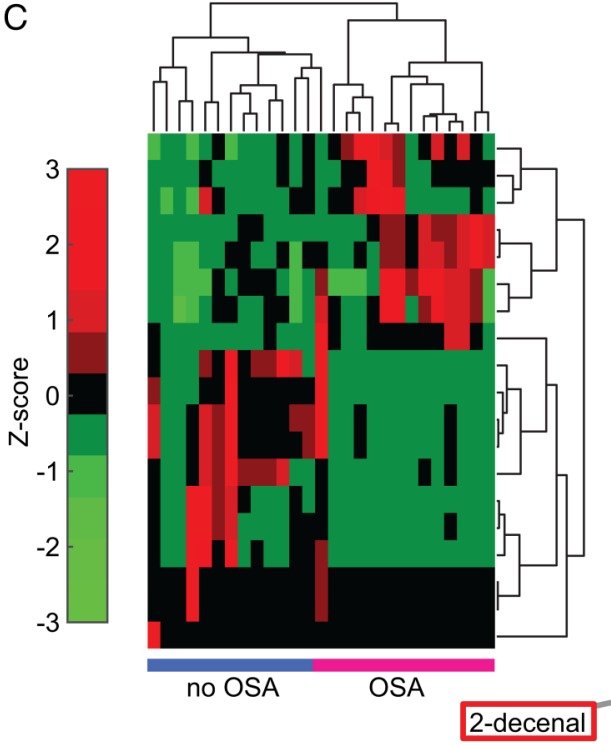

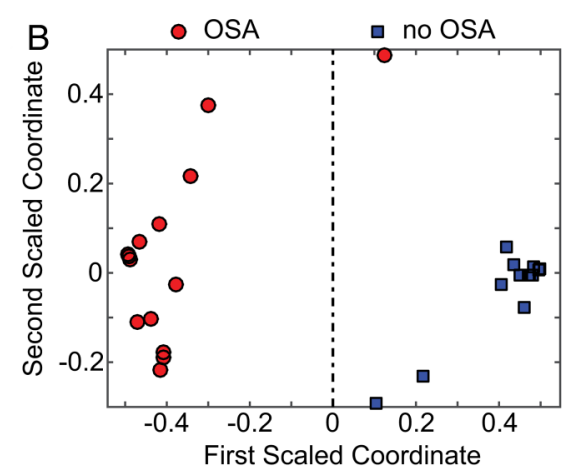

D<smiles>c1ccc2scnc2c1</smiles>

Experimental

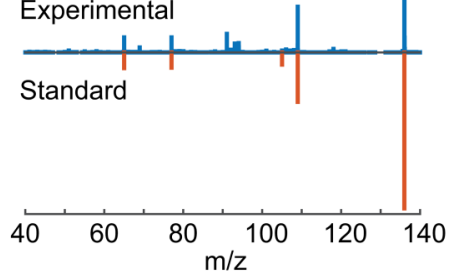

$\mathrm{E}$

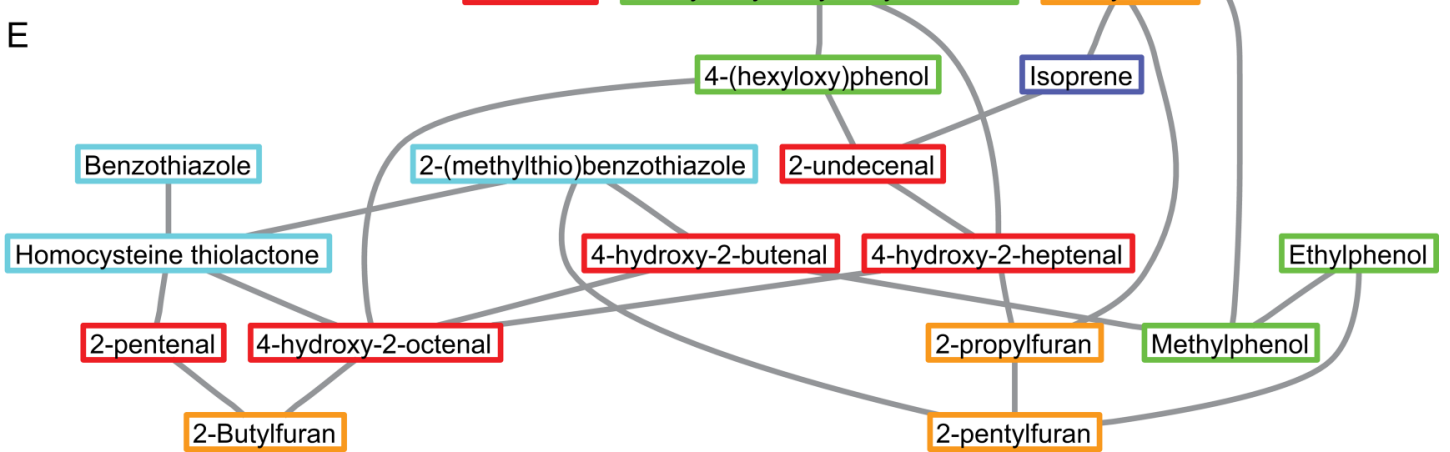

Figure 3 Obstructive sleep apnoea (OSA) prediction and metabolites identification are shown. (A) Receiver operating characteristic (ROC) curve obtained for OSA (ie, oxygen desaturation index (ODI) $>15 / h)$ prediction $(n=27)$; $(B)$ multidimensional scaling plot to the proximity matrix $(n=27)$ computed using the most frequently selected features visualising two distinct groups are shown. (C) Similarly, hierarchical cluster analysis $(n=27)$ revealed two distinct clusters; (D) head-to-tail fragmentation mass spectrum of benzothiazole found in breath (top) and the pure standard (bottom). (E) Visualisation of the associations of the identified metabolites. Interconnected metabolites are based on their partial correlations (requiring $\mathrm{p}<0.01$ ). Compounds in red frames are a family of aldehydes; in yellow frames a series of furans; in green frames phenolic compounds; in light blue frames sulphur-containing compounds; in dark blue others.

sensors. ${ }^{9} 10$ These studies have shown good discriminatory accuracy in OSA. However, electronic noses do not allow chemical analysis, limiting their use in elucidating pathophysiological mechanisms of diseases. State-of-the-art mass spectrometry has unparalleled chemical selectivity and sensitivity; however, most available commercial instruments were developed for offline analysis of liquid samples. Here we show that it is possible to take advantage of the powerful analytical capabilities of mass spectrometry, with minor hardware modifications that make instantaneous analysis of gas-phase metabolites in breath feasible. The used approach allows an immediate examination of breath with unparalleled chemical selectivity that (1) offers high diagnostic accuracy and at the same time (2) pinpoints the chemical structure of the exhaled molecules, with which a distinct metabolic fingerprint becomes recognisable. An additional natural advantage of examining metabolites in vivo is that any bias due to sample collection, storage and further manipulation is avoided. This is critical especially in the case of a gaseous sample such as breath.

In line with preliminary related work, we found that recurrence of OSA has a distinct altered metabolic breath profile. The technology used in this study can determine such metabolic breath profiles and make it available for detection of the disease (figure $3 \mathrm{~A}-\mathrm{C})$ within minutes $(<5 \mathrm{~min} /$ patient) (figure $2 \mathrm{~A}-\mathrm{D}$ ). Our data suggest that some metabolites are augmented in the breath of patients with OSA and also correlate with disease 
Table 4 Identified exhaled metabolites changing in response to recurrence of OSA

\begin{tabular}{|c|c|c|c|c|}
\hline \multicolumn{2}{|c|}{ Breath signal features } & \multicolumn{3}{|c|}{ Between-group changes $(n=26)$} \\
\hline $\mathrm{m} / \mathrm{z}$ & ID & $\mathrm{p}$ Value & q Value & $95 \% \mathrm{Cl}$ \\
\hline 69.0681 & Isoprene & 0.020 & 0.15 & 0.15 to 2.23 \\
\hline 97.0622 & 2-ethylfuran & 0.022 & 0.16 & 0.12 to 2.14 \\
\hline 102.0888 & 2-pentenal & 0.002 & 0.15 & 0.55 to 2.55 \\
\hline 109.0743 & Methylphenol & 0.002 & 0.15 & 0.58 to 2.57 \\
\hline 111.0782 & 2-propylfuran & 0.003 & 0.15 & 0.47 to 2.40 \\
\hline 118.0288 & Homocysteine thiolactone & 0.019 & 0.15 & 0.14 to 2.08 \\
\hline 123.0891 & Ethylphenol & 0.002 & 0.15 & 0.52 to 2.53 \\
\hline 125.0930 & 2-Butylfuran & 0.009 & 0.15 & 0.29 to 2.31 \\
\hline 129.0885 & 4-hydroxy-2-heptenal & 0.016 & 0.15 & 0.20 to 2.35 \\
\hline 136.0193 & Benzothiazole & 0.026 & 0.17 & 0.08 to 2.01 \\
\hline 139.1090 & 2-pentylfuran & 0.004 & 0.15 & 0.44 to 2.51 \\
\hline 155.1522 & 2-decenal & 0.016 & 0.15 & 0.15 to 1.79 \\
\hline 160.1298 & 4-hydroxy-2-octenal & 0.019 & 0.15 & 0.17 to 2.38 \\
\hline 166.0965 & Mevalonic acid & 0.032 & 0.17 & 0.04 to 1.70 \\
\hline 182.0068 & 2-(methylthio)benzothiazole & 0.020 & 0.15 & 0.13 to 1.99 \\
\hline 196.1102 & Digitalose & 0.007 & 0.15 & 0.32 to 2.34 \\
\hline
\end{tabular}

severity (ie, ODI; see figure $2 \mathrm{~F}$ and online supplementary figures E4 and E5). This suggests that these compounds may also be used to assess CPAP therapy effectiveness or compliance. Information derived from exhaled breath has the potential to characterise an individual's metabolic response to OSA which is important considering that to date, initiation of CPAP treatment mainly relies on symptoms and not on vascular risk, metabolic burden or comorbidities.

The fact that the most significant compounds identified belong to families of closely related molecules (eg, a homologous series of aldehydes) provides high confidence in the overall quality of the data obtained and implies that they are not raised merely by chance. This confidence is strengthened by the fact that the series of compounds for a given family correlate significantly with each other (see figure $3 \mathrm{E}$ and online supplementary figure E6). Most of the molecules identified here are known human metabolites related to different metabolic processes. For example a family of saturated aldehydes was significantly increased after CPAP withdrawal and correlate strongly with each other. Cell membrane lipid peroxidation leads to generation of various aldehydes. Subsequently, the released endproducts may trigger different cellular events. ${ }^{23}$ The closely related family of aldehydes found here reinforces the hypothesis of increased oxidative stress levels in OSA. ${ }^{24}$ Isoprene, one of the identified discriminating molecules correlating with undecenal, is part of the mevalonic cycle (cholesterol synthesis). ${ }^{25}$ Isoprene plays a role in sleep regulation and is increased in stressful conditions such as exercise, myocardial infarction and increased cardiac output. ${ }^{26-31}$ Increased isoprene in the exhaled breath of patients with OSA may be due to increased sympathetic activity. Furthermore, isoprenoids serve as a base frame for steroids and thus cortisol, and also play a role in lipid metabolism that is altered possibly because of oxidative stress in OSA. $^{32}$

Intriguingly, the family of aldehydes strongly correlates with a series of furans (see figure $3 \mathrm{E}$ and online supplementary figure E6). Furans have been found in the breath of healthy subjects.
These compounds are related to smoking and are also thought to be secondary metabolites from the gut microbiome. ${ }^{33} 34$ The number of former smokers in the CPAP withdrawal group was threefold higher than in the control group. However, this should not confound the finding of an increase in furans in the withdrawal group since it was only detected in response to CPAP withdrawal and was not present in the baseline breath analysis on therapeutic CPAP. The fact that only one participant in the withdrawal CPAP group was an active smoker suggests that the significant increase of alkyl furans in the CPAP withdrawal group is not related to active smoking but possibly to an altered gut flora in response to OSA recurrence, as found in a previous study. ${ }^{8}$ Similarly, gut microbiota metabolites have been reported to be altered in sleep-deprived humans. ${ }^{35}$ Along the same lines, intermittent hypoxia has been found to alter gut microbiota diversity in OSA mice models. ${ }^{36}$ Moreover, we also found a family of phenols, including cresol, which is a unique bacterial metabolite from protein fermentation. ${ }^{37}$ These metabolites are toxic bacterial end-products that, in the case of patients with compromised renal function, have been found to accumulate in the circulation, resulting in increased levels measurable in blood. ${ }^{38}$ Another gut microbiome-related identified compound is acetoin. ${ }^{34}$ Thus our data reinforce the notion that the altered metabolism associated with OSA may perturb the metabolic interplay between the gut flora and its host. In this regard, our findings add to the hypothesis that there is an association between gut microbiome composition and clinical phenotype. $^{39}$

Another molecule found to be augmented in the breath of patients with OSA is benzothiazole and its closely related metabolite 2-(methylthio)benzothiazole. Benzothiazole has previously been reported in breath. ${ }^{40}$ Interestingly, it has been found to be significantly increased in breath of patients with pulmonary arterial hypertension and cystic fibrosis. ${ }^{41}{ }^{42}$ It seems to correlate with pulmonary arterial pressure and pulmonary vascular resistance. ${ }^{41}$ The fact that it is significantly increased following CPAP withdrawal suggests that apnoea-related nocturnal hypoxaemia indeed is associated with increased pulmonary arterial pressure. However, benzothiazole derivatives are widely used industrial products and have been reported to occur in the environment (eg, drinking water). ${ }^{43}$ The reason why these exogenous compounds appear to be significantly altered in pulmonary hypertension, cystic fibrosis and OSA remains unknown. One hypothesis is that, as in the case of the toxic phenolic metabolites produced by gut bacteria, OSA could be associated with an impaired capacity to detoxify endogenous as well as exogenous small molecules, leading to an accumulation in blood and hence increased levels in breath. Another sulphur-containing molecule identified in the current study was homocysteine thiolactone, which has been associated with pathological conditions; for example, plasma levels have been linked with the development and progression of vascular complications in diabetic patients. ${ }^{44}$ In summary, our data support the association of OSA with increased sympathetic activity, oxidative stress and perturbation of gut microbiota-host equilibrium. $^{724}$

A possible limitation of our study is that the diagnostic accuracy of the OSA-specific exhaled breath pattern was not compared with polysomnography, which is considered the gold standard for diagnosis of OSA. Since this is a small sample of well-characterised patients, generalisability to other patients with OSA and a potential impact of comorbidities must be proven in future studies including more patients with OSA. As to the correlations found between breath signals and disease severity, one cannot completely exclude the possibility that the 
correlations are to some extent due to differences between groups. However, correlations matrices show a significant overlap between both groups indicating that there are true correlations between changes in disease severity and exhaled features. Another possible limitation of the study is that the role of potential confounders not related to the disease-for example, increased caffeine intake because of increased sleepiness during the 2-week study period-cannot be determined. However, the applied short-term CPAP withdrawal model comparing treated and untreated patients with OSA is less prone to general confounding factors that do not change in the short term (eg, body mass index among many others). To avoid the anticipated influence of breathing manoeuvres on exhaled compounds ${ }^{45}$ and to minimise artefacts, ${ }^{46}$ breath analysis was highly standardised including a constant exhalation pressure.

The exhaled compounds changing in response to CPAP withdrawal cannot be considered as disease-specific markers of OSA without caution before they are validated in a population of untreated patients with OSA. Furthermore, in future studies, exhaled breath compounds should be compared with metabolomics of other body sources such as blood (hybrid metabolomics). ${ }^{22}$

Nevertheless, the possibility of identifying exhaled metabolites in real time and with high chemical selectivity is of general interest and makes this technique attractive for use in research and possibly in clinical practice. As such exhaled breath metabolomics can be applied for diagnostic goals, therapy guidance and monitoring, and to provide clues to underlying mechanisms.

In conclusion, real-time exhaled breath analysis by SESI-MS allows rapid and accurate detection of OSA recurrence. The technique has the potential to characterise an individual's metabolic response to OSA and thus makes a comprehensible phenotyping of OSA possible. However, to draw definite conclusions on diagnostic accuracy, the findings of this study have to be validated in an untreated OSA population.

Contributors Conception and design: MK, EIS, KEB, PM-LS, JRS and RZ. Data acquisition: EIS, LB, TG, DGG, NS and YN-O. Analysis and interpretation of data: PM-LS, EIS, DGG, MTG and YS. Drafting the article: EIS, PM-LS and MK. Revising the article for important intellectual content and final approval: all authors.

Funding This trial was supported by grants from the Swiss National Science Foundation (CR23I2_149617 and 32003B_143365/1), the University of Zurich Clinical Research Priority Program Sleep and Health and a Marie Curie European Reintegration Grant within the 7th European Community Framework Programme (276860).

Competing interests None declared

Ethics approval Cantonal Ethics Committee of Zurich.

Provenance and peer review Not commissioned; externally peer reviewed.

\section{REFERENCES}

1 Peppard PE, Young T, Barnet JH, et al. Increased prevalence of sleep-disordered breathing in adults. Am J Epidemiol 2013;177:1006-14.

2 Young T, Evans L, Finn L, et al. Estimation of the clinically diagnosed proportion of sleep apnea syndrome in middle-aged men and women. Sleep 1997;20:705-6.

3 George CF. Reduction in motor vehicle collisions following treatment of sleep apnoea with nasal CPAP. Thorax 2001;56:508-12.

4 Kohler M, Stoewhas AC, Ayers L, et al. Effects of continuous positive airway pressure therapy withdrawal in patients with obstructive sleep apnea: a randomized controlled trial. Am J Respir Crit Care Med 2011;184:1192-9.

5 Marin JM, Carrizo SJ, Vicente E, et al. Long-term cardiovascular outcomes in men with obstructive sleep apnoea-hypopnoea with or without treatment with continuous positive airway pressure: an observational study. Lancet 2005;365:1046-53.

6 Lévy P, Bonsignore MR, Eckel J. Sleep, sleep-disordered breathing and metabolic consequences. Eur Respir J 2009;34:243-60.

7 Kohler M, Stradling JR. Mechanisms of vascular damage in obstructive sleep apnea. Nat Rev Cardiol 2010;7:677-85.
8 Ferrarini A, Rupérez FJ, Erazo M, et al. Fingerprinting-based metabolomic approach with LC-MS to sleep apnea and hypopnea syndrome: a pilot study. Electrophoresis 2013;34:2873-81.

9 Benedek P, Lázár Z, Bikov A, et al. Exhaled biomarker pattern is altered in children with obstructive sleep apnoea syndrome. Int I Pediatr Otorhinolaryngol 2013;77:1244-7.

10 Greulich T, Hattesohl A, Grabisch A, et al. Detection of obstructive sleep apnoea by an electronic nose. Eur Respir J 2013:42:145-55.

11 Aernecke MJ, Mendum T, Geurtsen G, et al. Vapor pressure of Hexamethylene Triperoxide Diamine (HMTD) estimated using secondary electrospray ionization mass spectrometry. J Phys Chem A 2015;119:11514-22.

12 Zhu J, Bean HD, Jiménez-Díaz J, et al. Secondary electrospray ionization-mass spectrometry (SESI-MS) breathprinting of multiple bacterial lung pathogens, a mouse model study. J Appl Physiol (1985) 2013;114:1544-9.

13 Dillon LA, Stone VN, Croasdell LA, et al. Optimisation of secondary electrospray ionisation (SESI) for the trace determination of gas-phase volatile organic compounds. Analyst 2010;135:306-14.

14 Wu C, Siems WF, Hill HH, Jr. Secondary electrospray ionization ion mobility spectrometry/mass spectrometry of illicit drugs. Anal Chem 2000;72:396-403.

15 Martinez-Lozano Sinues P, Landoni E, Miceli R, et al. Secondary electrospray ionization-mass spectrometry and a novel statistical bioinformatic approach identifies a cancer-related profile in exhaled breath of breast cancer patients: a pilot study. J Breath Res 2015;9:031001.

16 Martinez-Lozano Sinues P, Meier L, Berchtold C, et al. Breath analysis in real time by mass spectrometry in chronic obstructive pulmonary disease. Respiration 2014:87:301-10

17 Schwarz El, Schlatzer C, Stehli J, et al. The effects of short-term CPAP withdrawal on myocardial perfusion in OSA - a randomised placebo-controlled trial. Eur Respir J 2014;44(Suppl 58): 4664

18 Storey JD. A direct approach to false discovery rates. J $R$ Statist Soc $B$ 2002;64:479-98.

19 Breiman L. Random forests. Mach Learn 2001;45:5-32.

20 Feliciano A, Torres VM, Vaz F, et al. Overview of proteomics studies in obstructive sleep apnea. Sleep Med 2015;16:437-45.

21 Maeder MT, Strobel W, Christ M, et al. Comprehensive biomarker profiling in patients with obstructive sleep apnea. Clin Biochem 2015;48:340-6.

22 Broza YY, Mochalski $\mathrm{P}$, Ruzsanyi $\mathrm{V}$, et al. Hybrid volatolomics and disease detection. Angew Chem Int Ed Engl 2015;54:11036-48.

23 Rahman I. Role of oxidative stress and inflammatory response in smokers and chronic obstructive pulmonary disease. Oxidative Stress, Inflammation, and Health: CRC Press, 2005:291-368.

24 Lavie L. Oxidative stress inflammation and endothelial dysfunction in obstructive sleep apnea. Front Biosci (Elite Ed) 2012;4:1391-403.

25 Deneris ES, Stein RA, Mead JF. In vitro biosynthesis of isoprene from mevalonate utilizing a rat liver cytosolic fraction. Biochem Biophys Res Commun 1984;123:691-6.

26 DeMaster EG, Nagasawa HT. Isoprene, an endogenous constituent of human alveolar air with a diurnal pattern of excretion. Life Sci 1978:22:91-7.

27 Cailleux A, Allain P. Isoprene and sleep. Life Sci 1989;44:1877-80.

28 King J, Kupferthaler A, Frauscher B, et al. Measurement of endogenous acetone and isoprene in exhaled breath during sleep. Physiol Meas 2012;33:413-28.

29 Karl T, Prazeller P, Mayr D, et al. Human breath isoprene and its relation to blood cholesterol levels: new measurements and modeling. J App/ Physiol 2001;91:762-70

30 Mendis S, Sobotka PA, Euler DE. Expired hydrocarbons in patients with acute myocardial infarction. Free Radic Res 1995;23:117-22

31 Pabst F, Miekisch W, Fuchs $\mathrm{P}$, et al. Monitoring of oxidative and metabolic stress during cardiac surgery by means of breath biomarkers: an observational study. I Cardiothorac Surg 2007;2:37.

32 Lavie L, Vishnevsky A, Lavie P. Evidence for lipid peroxidation in obstructive sleep apnea. Sleep 2004;27:123-8.

33 Ulanowska A, Kowalkowski T, Trawińska E, et al. The application of statistical methods using VOCs to identify patients with lung cancer. J Breath Res 2011;5:046008.

34 Garner CE, Smith S, de Lacy Costello B, et al. Volatile organic compounds from feces and their potential for diagnosis of gastrointestinal disease. FASEB J 2007;21:1675-88.

35 Weljie AM, Meerlo P, Goel N, et al. Oxalic acid and diacylglycerol 36:3 are cross-species markers of sleep debt. Proc Natl Acad Sci USA 2015;112: 2569-74.

36 Moreno-Indias I, Torres M, Montserrat JM, et al. Intermittent hypoxia alters gut microbiota diversity in a mouse model of sleep apnoea. Eur Respir J 2015:45:1055-65.

37 Hamer HM, De Preter V, Windey K, et al. Functional analysis of colonic bacterial metabolism: relevant to health? Am J Physiol Gastrointest Liver Physiol 2012;302: G1-9.

38 Evenepoel P, Meijers BKI, Bammens BRM, et al. Uremic toxins originating from colonic microbial metabolism. Kidney Int 2009;76:S12-19. 
39 Le Chatelier E, Nielsen T, Qin J, et al. Richness of human gut microbiome correlates with metabolic markers. Nature 2013;500:541-6.

40 Phillips $\mathrm{M}$, Herrera J, Krishnan S, et al. Variation in volatile organic compounds in the breath of normal humans. J Chromatogr B Biomed Sci Appl 1999;729:75-88.

41 Mansoor JK, Schelegle ES, Davis CE, et al. Analysis of volatile compounds in exhaled breath condensate in patients with severe pulmonary arterial hypertension. PLOS ONE 2014;9.

42 Robroeks CMHHT, van Berkel JJBN, Dallinga JW, et al. Metabolomics of volatile organic compounds in cystic fibrosis patients and controls. Pediatr Res 2010:68:75-80.
43 Asimakopoulos AG, Wang L, Thomaidis NS, et al. Benzotriazoles and benzothiazoles in human urine from several countries: a perspective on occurrence, biotransformation, and human exposure. Environ Int 2013;59:274-81.

44 Jakubowski H, Głowacki R. Chemical Biology of Homocysteine Thiolactone and Related Metabolites. Adv Clin Chem, 2011;55:81-103.

45 Sukul P, Trefz P, Schubert JK, et al. Immediate effects of breath holding maneuvers onto composition of exhaled breath. J Breath Res 2014;8:037102.

46 Thekedar B, Oeh U, Szymczak W, et al. Influences of mixed expiratory sampling parameters on exhaled volatile organic compound concentrations. I Breath Res 2011:5:016001. 